\title{
NM23 as a prognostic biomarker in ovarian serous carcinoma
}

\author{
Bo Sung Youn ${ }^{1}$, Dong-Su Kim², Jae Wook Kim³ , Young Tae Kim³ ${ }^{3}$ Suki Kang ${ }^{4}$ and \\ Nam Hoon $\mathrm{Cho}^{4}$ \\ ${ }^{1}$ Department of Obstetric Gynecology, Kangnam CHA Hospital, Seoul, Korea; ${ }^{2}$ Genomine Research Division, \\ Genomine Inc., Pohang, Korea; ${ }^{3}$ Department of Obstetric Gynecology, Yonsei University College of Medicine, \\ Seoul, Korea and ${ }^{4}$ Department of Pathology, Yonsei University College of Medicine, Seoul, Korea
}

\begin{abstract}
The $n$ m23 gene is a reported metastasis suppressor gene. Recent studies have shown that its expression has tissue specificity. The role of $n m 23$ in human ovarian cancer is still controversial. This study examines the prognostic significance of $n \mathbf{m} 23$ expression in patients with serous ovarian carcinoma. Following comparative proteomics in 13 fresh frozen ovarian serous cancer tissues with other histological types of ovarian cancers, validation was performed using immunohistochemistry on clinically well-designed 73 ovarian serous carcinoma microarray samples that were retrieved from ovarian cancer patients from 1990 to 2003 . Statistical analysis of the results was performed using $\chi^{2}$ test, Cox proportional regression, the Kaplan-Meier method and log-rank test. We found that the expression of $n m 23$ inversely correlated with peritoneal seeding $(P=0.009)$. However, strong $n m 23$ expression was associated with mortality in patients with ovarian carcinoma in univariate analysis $(P=\mathbf{0 . 0 4})$. Poor prognostic factors of disease-free survival included tumor residue more than $2 \mathrm{~cm}(P=0.02)$, bilaterality $(P=0.01)$ and peritoneal seeding $(P<0.01)$, whereas poor prognostic factors affecting overall survival included peritoneal seeding $(P=0.05)$. In Kaplan-Meier analysis, strong $n m 23$ immunoreactivity correlates with poor overall survival $(P=0.04)$ but not with poor disease-free survival. In conclusion, overexpression of $n m 23$ is independently associated with decreased overall survival in patients with ovarian carcinoma and also significantly correlates with mortality. $\mathrm{Nm} 23$ may have a biological function that leads to poor clinical outcomes in ovarian carcinoma.
\end{abstract}

Modern Pathology (2008) 21, 885-892; doi:10.1038/modpathol.2008.64; published online 11 April 2008

Keywords: $n$ m23; proteomics; serous ovarian carcinoma; cancer metastasis

The lethality of serous ovarian carcinomas is primarily attributed to its advanced stage at the time of initial diagnosis. It spreads rapidly along the peritoneum and omentum in approximately $70 \%$ of patients and eventually metastasizes. ${ }^{1}$ Despite advances in cytotoxic therapies, only $30 \%$ of ovarian cancer patients survive 5 years after their initial diagnosis. ${ }^{1-3}$ Therefore, understanding the molecular mechanisms regulating the invasion or metastatic behavior of ovarian cancer will likely have a significant impact on the outcomes of this devastating disease and/or on the diagnosis of early-stage ovarian cancer in patients who are at risk for subsequent metastatic progression or relapse.

Correspondence: Professor NH Cho, MD, Department of Pathology, Yonsei University College of Medicine, SeodaemunkuSinchon dong 142, Seoul 120-752, Republic of Korea.

E-mail: cho1988@yumc.yonsei.ac.kr

Received 15 January 2008; revised 13 February 2008; accepted 14

February 2008; published online 11 April 2008
In general, cancer metastasis is an active process involving multiple, sequential cellular events that are regulated by numerous genes. In contrast, ovarian carcinoma metastasis is the result of a relatively passive process. ${ }^{2}$ Once a tumor disrupts the ovarian capsule, it may grow so large that it extends to and makes contact with the omentum. Alternatively, cells from the primary tumor may be transported to the omentum via the peritoneal fluid. ${ }^{2}$ Previous genomic studies have shown that there are little differences in gene expression profiles between ovarian carcinomas and their omentum metastases. ${ }^{3}$

We have previously used a comparative proteomic approach and various other subtractive and comparative methods to examine the gene expression profiles of ovarian carcinomas. ${ }^{4}$ The results of these studies have led to the identification of several putative biomarkers for ovarian carcinomas. These candidate genes included nm23, annexin I, protein phosphatase-1, ferritin, proteasome $\alpha-6$, N-acetyl glucosamine kinase (NAGK) and an A6-related 
protein. ${ }^{4}$ Interestingly, we found that the $n m 23 / N D P$ kinase family is typically overexpressed in ovarian cancers but generally underexpressed in metastatic cells. In the current study, we use proteomics and immunohistochemistry to examine the biological role of nm23 in serous ovarian carcinomas.

\section{Materials and methods}

\section{Tissue Specimens and Sample Preparation for 2E}

Fresh frozen samples of 13 ovarian serous cancer tissues were obtained from the Yonsei University College of Medicine (Table 1). Fresh frozen tissues were prepared from eight other types of ovarian

Table 1 Correlation of nm23 expression and clinical variables

\begin{tabular}{|c|c|c|}
\hline Variables & $n m 23$ & $\mathrm{P}$-value \\
\hline Age (years) & & 0.51 \\
\hline$<50$ & 42 & \\
\hline$\geq 50$ & 35 & \\
\hline Stage & & 0.06 \\
\hline I, II & 53 & \\
\hline III, IV & 24 & \\
\hline Grade & & 0.12 \\
\hline $1-2$ & 30 & \\
\hline 3 & 47 & \\
\hline Ascites & & 0.97 \\
\hline Negative & 40 & \\
\hline Positive & 37 & \\
\hline CA125 level (U/ml) & & 0.76 \\
\hline$<35$ & 13 & \\
\hline $35-100$ & 23 & \\
\hline$>100$ & 41 & \\
\hline Residual volume & & 0.40 \\
\hline Optimal & 43 & \\
\hline Non-optimal & 34 & \\
\hline Ovarian bilateralness & & 0.80 \\
\hline Unilateral & 40 & \\
\hline Bilateral & 37 & \\
\hline Peritoneal seeding & & $0.009^{\mathrm{a}}$ \\
\hline Negative & 60 & \\
\hline Positive & 17 & \\
\hline LN metastasis & & 0.28 \\
\hline Negative & 48 & \\
\hline Positive & 29 & \\
\hline Distant metastasis & & 0.12 \\
\hline Negative & 19 & \\
\hline Positive & 58 & \\
\hline Dead status & & $0.04^{\mathrm{a}}$ \\
\hline Alive & 25 & \\
\hline Dead & 52 & \\
\hline Recurrent status & & 0.09 \\
\hline No recurrence & 29 & \\
\hline Recurrence & 48 & \\
\hline
\end{tabular}

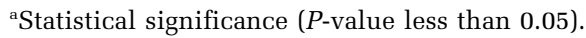

cancers (five endometrioid cancers and three mucinous cancers) and three samples of kidney, liver, lung and cervical cancer tissues. All samples were prepared with the consent of the patients who donated tissues.

\section{IPG 2D PAGE}

IPG dry strips were equilibrated for $12-16 \mathrm{~h}$ in a solution containing $7 \mathrm{M}$ urea, $2 \mathrm{M}$ thiourea containing 2\% 3-[(3-cholamidopropy) dimethylammonio]1-propanesulfonate, $1 \%$ dithiothreitol (DTT) and $1 \%$ pharmalyte. The equilibrated strips were loaded with $200 \mu \mathrm{g}$ of sample. Isoelectric focusing (IEF) was performed at $20^{\circ} \mathrm{C}$ using a Multiphor II electrophoresis unit and an EPS 3500 XL power supply (Amersham Biosciences) following the manufacturer's instructions. For IEF, the voltage was linearly increased from 150 to $3500 \mathrm{~V}$ over $3 \mathrm{~h}$ (to allow for sample entry), then the voltage was held constant at $3500 \mathrm{~V}$ with the focusing complete after $96 \mathrm{kVh}$. Prior to the second dimension, the strips were incubated for $10 \mathrm{~min}$ in equilibration buffer (50 mM Tris-Cl, pH 6.8 containing $6 \mathrm{M}$ urea, $2 \%$ SDS and $30 \%$ glycerol) with $1 \%$ DTT and $2.5 \%$ iodoacetamide. The equilibrated strips were then inserted onto SDS-PAGE gels $(20-24 \mathrm{~cm}, 10-16 \%)$ that were run using a Hoefer DALT 2D system (Amersham Biosciences) following the manufacturer's instructions. The $2 \mathrm{D}$ gels were run at $20^{\circ} \mathrm{C}$ for $1700 \mathrm{Vh}$, then silver stained by the fixing and sensitization steps with glutaraldehyde being omitted.

\section{Image Analysis}

Quantitative analyses of the digitized images were performed using the PDQuest software (version 7.0; BioRad) according to the protocols provided by the manufacturer. The quantity of each spot was normalized to the total valid spot intensity. Protein spots were identified as having a significant variation in expression if their expression level deviated by more than twofold relative to the expression level detected in the control or normal sample.

\section{MALDI-TOF MS}

A total of 64 gel spots (nucleus: 31, cytoplasm: 33) were excised from preparative gels using biopsy punches and transferred to a $1.5 \mathrm{ml}$ siliconized Eppendorf tube. The gel spots were destained in destaining solution containing $100 \mathrm{mM} \mathrm{Na} \mathrm{S}_{2} \mathrm{O}_{3}$ (Sigma) and $30 \mathrm{mM} \mathrm{K}_{3} \mathrm{Fe}(\mathrm{CN})_{6}$ (Sigma) (V/V, 1:1). Destained gel pieces were pre-reduced with $100 \%$ acetonitrile (HPLC grade). Then the protein-containing gel spots were reduced in reduction buffer $\left(100 \mathrm{mM} \quad \mathrm{NH}_{4} \mathrm{HCO}_{3}\right.$ (Sigma) and $10 \mathrm{mM}$ DTT (Sigma)) for $30 \mathrm{~min}$ at $56^{\circ} \mathrm{C}$ and then alkylated in 
alkylation buffer (100 $\mathrm{mM} \mathrm{NH} \mathrm{NHCO}_{3}$ (Sigma) and $55 \mathrm{mM}$ iodoacetamide (Sigma)) in the dark for $25 \mathrm{~min}$ at room temperature. The gel pieces were dried using a Speed-Vac. The dried gel pieces were incubated in $\mathrm{ABC}$ buffer $(50 \mathrm{mM}, \mathrm{pH}$ 8.0) containing $0.1 \mu \mathrm{g} / \mu \mathrm{l}$ trypsin for $12-16 \mathrm{~h}$ at $37^{\circ} \mathrm{C}$. Following concentration, the peptide mixture was desalted using $\mathrm{C}_{18}$ ZipTips (Millipore), and the peptides were eluted in $1-5 \mu \mathrm{l}$ of $50 \%$ ACN/ $0.1 \%$ TFA. An aliquot of this solution was mixed with an equal volume of a saturated solution of $\alpha$-cyano-4-hydroxycinnamic acid (Sigma) in $50 \% \mathrm{ACN} / 0.1 \% \mathrm{TFA}$, and $1 \mu \mathrm{l}$ of this mixture was spotted onto a target plate. The tryptic peptide mixture was extracted and purified using a Millipore ZipTip C18 column and concentrated using ZipTips (Millipore Corp.). The peptide samples were cocrystallized with matrix on the Voyager $96 \times 2$ sample plate $(\mathrm{P} / \mathrm{N}$ V700813) using $0.5 \mu \mathrm{l}$ matrix ( $\alpha$-cyano-4-hydroxytranscinnamic acid) and $0.5 \mu \mathrm{l}$ sample. The samples were then analyzed using the Applied Biosystems Voyager System 4307 MALDI-TOF Mass Spectrometer (ABI), and desArg $^{1}$-Bradykinin, Glu ${ }^{1}$-Fibrinopeptide B and ACTH (clip 18-39) served as external standards for the mass calibration.

\section{Clinical Survey for Tested Patients}

We examined the medical records and archival slides in the collection of ovarian serous adenocarcinoma in the Gynecologic Oncology Files of Yonsei University College of Medicine in Korea. We selected 73 cases of ovarian serous carcinomas since 1990 for further review. Demographics (including age, sex, tumor site, tumor size, number of tumors, frequency of tumor recurrence and progression, associated malignancy and survival time) were obtained from each patient's medical records. The time to recurrence was calculated from the time of complete response (ie, no evidence of disease) to the time of the first tumor recurrence upon follow-up examination. All patients underwent total abdominal hysterectomy with both salpingo-oophorectomy and pelvic lymphadenectomy, including omentectomy. Patients with advanced ovarian cancer (stage III/IV) were also given postoperative chemotherapy.

The medical records of all tested patients were surveyed for the following information: age, stage of carcinoma, grade, ascites with positive peritoneal cytology, preoperative serum CA 125, postoperative tumor residue $(2 \mathrm{~cm})$, bilaterality, peritoneal implants, lymph node involvement and distant metastasis. The staging was in accordance with the standards of the International Federation of Gynecology and Obstetrics. Optimal cytoreduction was defined as less than $2 \mathrm{~cm}$ of postoperative tumor residue. Disease-specific survival was calculated using the Kaplan-Meier method, and statistical analyses were performed using the log-rank test. The Cox proportional hazards model was used for multivariate parameter analyses, and a stepwise model was used to select independent prognostic factors. A $P$-value of less than 0.05 was considered to be statistically significant. All statistical data were analyzed using SPSS (SPSS ver. 12.0; Chicago, IL, USA).

\section{Tissue Specimens and Preparation of Samples for Immunohistochemistry}

Seventy-three samples of ovarian serous carcinomas isolated from 1990 to 2003 were available for immunohistochemical analysis and censored data. Recipient blocks were made from purified agar in $3.8 \times 2.2 \times 0.5 \mathrm{~cm}$ frames. Using a core needle, $2-\mathrm{mm}$ holes were made in these recipient blocks, and the resulting agar cores were discarded. The paraffin donor blocks were prepared following a thorough evaluation of the hematoxylin-eosin-stained slides. Two adjacent areas of a carcinoma from the matching donor blocks were transplanted to the recipient blocks using a 2-mm core needle. We constructed an array of samples of adjacent normal areas from these patients using paraffin-embedded, formalin-fixed tissue blocks. The recipient blocks were framed in the same mold that was used for the conventional paraffin block. Paraffin was then added to the frame. Consecutive $4-\mu \mathrm{m}$ thick sections were cut from the recipient blocks using an adhesive-coated slide system (Instrumedics Inc., Hackensack, NJ, USA).

The $4-\mu \mathrm{m}$ sections were placed on silane-coated slides, deparaffinized, immersed in phosphate-buffered saline (PBS) containing $0.3 \%(\mathrm{v} / \mathrm{v})$ hydrogen peroxide and processed in a microwave oven (in $10 \mathrm{mM}$ sodium citrate buffer, $\mathrm{pH} 6.5$, for $15 \mathrm{~min}$ at $700 \mathrm{~W})$. After blocking with $1 \%(\mathrm{w} / \mathrm{v})$ bovine serum albumin in PBS containing $0.05 \%(\mathrm{v} / \mathrm{v})$ Tween 20 for $30 \mathrm{~min}$, the slides were incubated overnight at $4^{\circ} \mathrm{C}$ with nm23. Immunoperoxidase staining was performed using the streptavidin-biotin peroxidase complex method (LSAB universal kit; DAKO, Carpinteria, CA, USA). As negative controls, subtype-matched normal mouse IgGs were used. The final reaction product was visualized with the addition of $0.03 \%(\mathrm{w} / \mathrm{v})$ of $3,3^{\prime}$-diaminobenzidine tetrachloride for 5-20 min. Strong cytoplasmic staining was considered a positive result. Immunostaining was graded and scored as follows: 0 (no staining), $1+$ (weak, diffuse staining) and $2+$ (strong, diffuse staining).

\section{Database Analysis}

The MS-Fit (UCSF Mass Spectrometry, ProteinProspector $\mathrm{v}$ 4.0.5) was used for protein identification via peptide mass fingerprinting. The spectra were calibrated using trypsin autodigestion ion peaks $\mathrm{m} / \mathrm{z}$ $(842.510,2211.1046)$ as internal standards. SwissProt. 10.30.2003 and NCBInr. 10.21.2003 were used as databases. 


\section{Results}

\section{Proteome Analysis of Human Ovarian Tumor Tissues}

The 2D-E protein patterns were quantified and matched. To select protein variations, the protein patterns of the tumor and control samples were divided into two classes, and the quantities of all of the detected spots in each class were compared using the PDQuest software ( $v$ 7.0). To compare the differences in protein expression specifically in ovarian tumors, we included data from other tumors, including lung cancer, hepatoma, cervical cancer and renal cell carcinoma. The PMF maps were then obtained using MALDI-TOF MS. These data were used to search MS-Fit (ProteinProspector $\mathrm{v}$ 4.0.5), and the identity of protein was determined by comprehensively considering the corresponding experimental MOWSE score, the number of matched peptides Mp PI and the sequence coverage. Comparisons of spot expressions in the tissues analyzed revealed that all of the tumors tested (kidney, lung, uterine cervix and ovary), with the exception of hepatoma, had significantly higher $n m 23$ expression than their corresponding normal tissues. The $n m 23$ was more highly expressed in serous and endometrioid types of ovarian cancer and was more weakly expressed in mucinous types of ovarian cancer (Figure 1).

\section{Clinical Correlation of nm23 Immunoreactivity}

Ovarian serous carcinomas had positive immunoreactivity for $\mathrm{nm} 23$ (Figure 2). The clinical correlation with $n m 23$ expression is illustrated in Table 1. The strong expression of $n m 23$ was inversely related to the presence of peritoneal implants. Specifically, $60 \%$ of the patients with strong $n m 23$ expression had no peritoneal implants, whereas only $27 \%$ of those patients had peritoneal implants $(P=0.009)$. In addition, a stronger level of $n m 23$ expression was detected in patients with higher stages (III/IV) of disease than in those with lower stages (I/II) of the disease, but these differences are not significant $(P=0.06)$. Nm23 overexpression was significantly more frequent in dead patients of ovarian cancer than in alive patients (54 vs $28 \% ; P=0.02$ ).

\section{Survival Analysis and nm23 Expression in Ovarian Cancer}

Multiple parameters have been used to predict the biological behavior of ovarian serous cancer by univariate (Table 2) and multivariate survival analyses (Table 3). In multivariate analysis, poor prognostic factors of disease-free survival included tumor residue more than $2 \mathrm{~cm}(P=0.02)$, bilaterality $(P=0.01)$ and peritoneal seeding $(P<0.01)$, whereas poor prognostic factor affecting overall survival included peritoneal seeding $(P=0.05)$. In KaplanMeier analysis, strong $n m 23$ immunoreactivity correlates with poor overall survival $(P=0.04)$ but not with poor disease-free survival (Figure 3 ).

\section{Discussion}

Using proteomics and immunohistochemistry, we discovered that $n m 23$ overexpression correlates with distant metastasis and reduced disease-free and overall survival in ovarian serous carcinoma, whereas reduced $n m 23$ expression is strongly

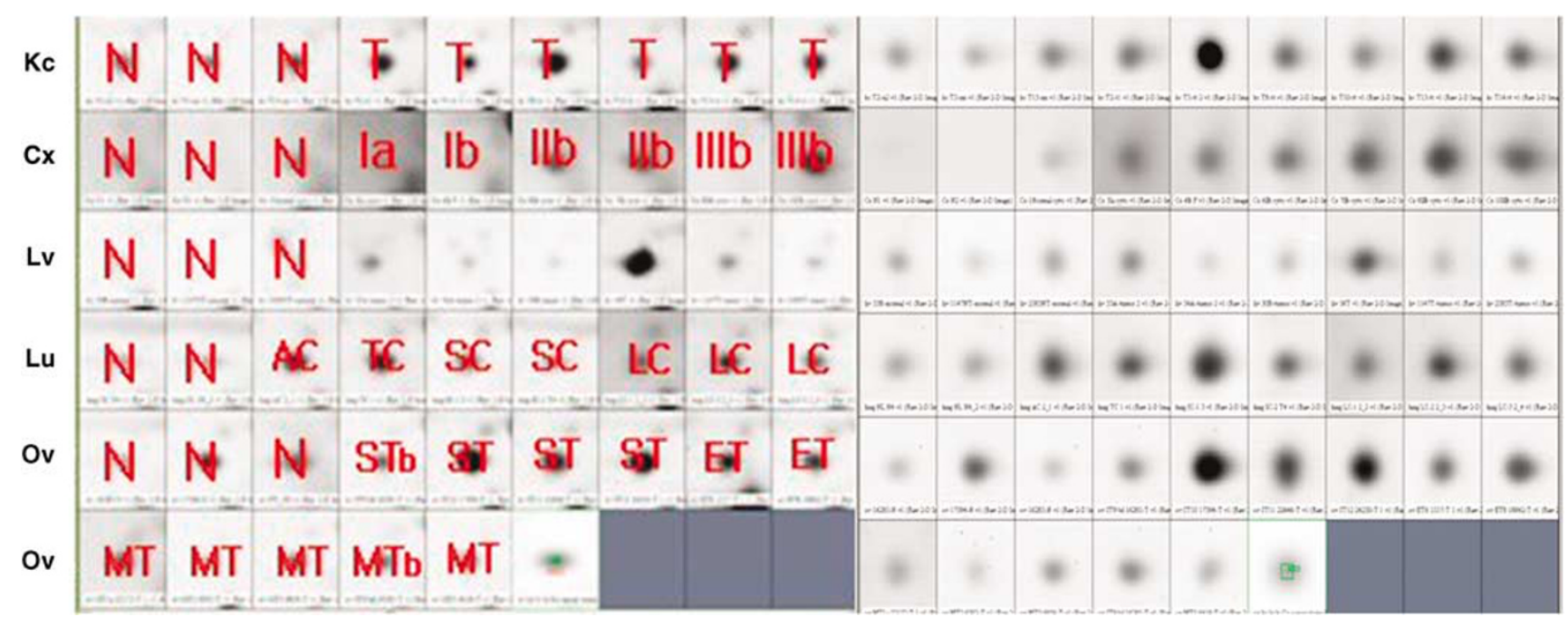

Figure 1 Comparative quantification in 2D-E protein spot. This spot was confirmed to be $n m 23 A$ expressed in isoform A using MS-Fit. The above figure represents legend of each row and column corresponding to the below figure. Notice the strongest spot in ovarian serous carcinoma relative to endometrioid and mucinous tumors. There is an increase in $n m 23$ expression in cervical cancer, lung cancer and renal cell carcinoma, whereas there is a decrease in nm23 expression in hepatoma. Cx, cervical cancer; Kc, kidney cancer; Lu, lung cancer; Lv, liver cancer; N, normal; Ov, ovary cancer; T, tumor. Each roman number in the Cx (cervical cancer) represents FIGO stage. ET,endometrioid carcinoma; MT, mucinous carcinoma; ST, serous carcinoma; STb, serous tumor of borderline malignancy. 

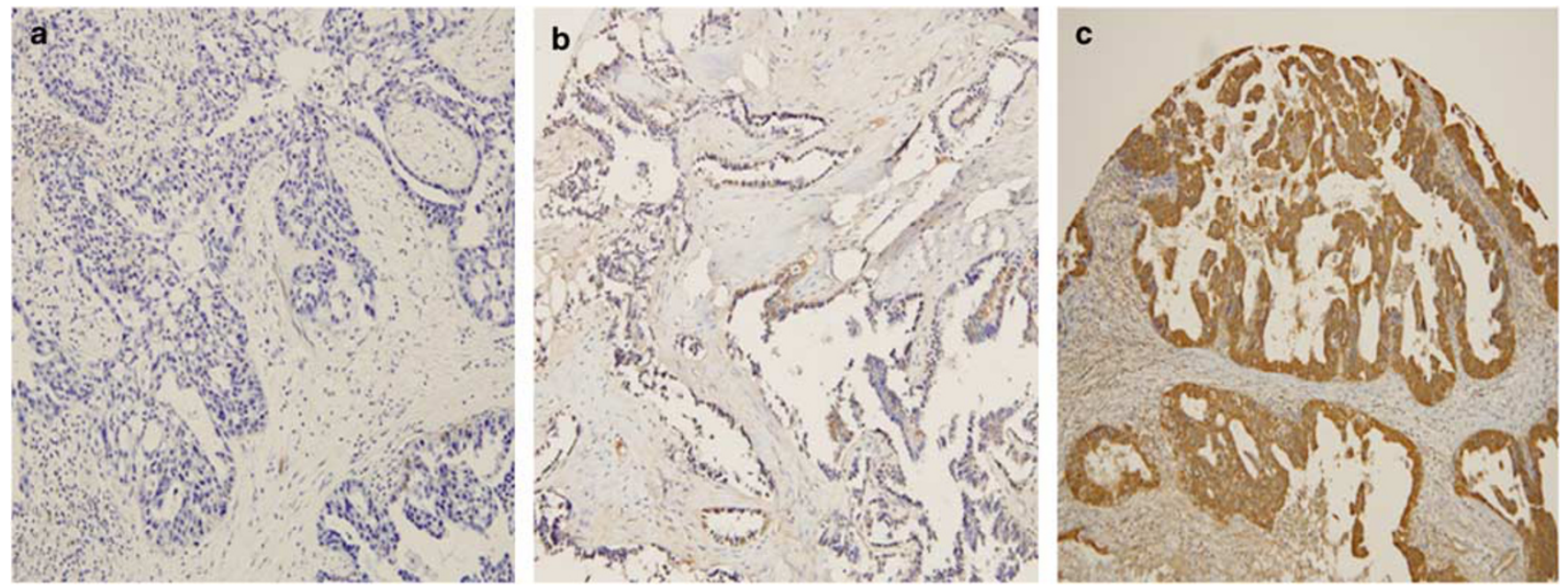

Figure 2 Immunohistochemical results of nm23 in ovarian serous adenocarcinomas. (a) Negative immunostaining for $n m 23$. (b) Focal, weak staining for nm23, graded as $1+$. (c) Intense, strong immunoreactivity for $n m 23$, graded as $2+$ (which is regarded as a true positive result).

Table 2 Univariate survival analysis of nm23 overexpression in ovarian serous carcinoma

\begin{tabular}{lccc}
\hline Variables & Median survival (years) & HR (95\% CI) & P-value \\
\hline $\begin{array}{l}\text { Nm23 } \\
\text { Negative } \\
\text { Positive }\end{array}$ & Not reached & $1.30(1.01-1.68)$ & $0.04^{*}$ \\
& 4.50 & & \\
& Disease-free survival (years) & $H R(95 \%$ CI) & P-value \\
\hline $\begin{array}{l}\text { Nm23 } \\
\text { Negative } \\
\text { Positive }\end{array}$ & 2.00 & $1.17(0.96-1.42)$ & 0.11 \\
\hline
\end{tabular}

*Statistical significance ( $P$-value less than 0.05$)$.

Table 3 Multivariate survival analysis of nm23 overexpression in ovarian serous carcinoma

\begin{tabular}{lcr}
\hline Variables & HR (95\% CI) & P-value \\
\hline Overall survival & & \\
$\quad$ Peritoneal seeding & $47.60(1.04-2421.7)$ & $0.05^{\mathrm{a}}$ \\
& & \\
Disease-free survival & & \\
$\quad$ Residual volume: non-optimal & & \\
Ovarian bilateralness & $2.09(1.13-3.87)$ & $0.02^{\mathrm{a}}$ \\
Peritoneal seeding & $3.36(1.31-8.62)$ & $0.01^{\mathrm{a}}$ \\
\hline
\end{tabular}

${ }^{\mathrm{a}}$ Statistical significance.

${ }^{\mathrm{b}}$ Non-optimal: residual volume $\geq 2 \mathrm{~cm}$.

associated with peritoneal implants. On the basis of these results, we propose that the differential expression of $n m 23$ affects its biological role. The association of $n m 23$ overexpression with distant metastasis is surprising, given the traditional view of $n m 23$ as a metastasis suppressor gene. Although this finding is contradictory, it is meaningful because it represents the first analysis of the role of $n m 23$ in ovarian cancer tissue samples using a proteomics approach involving well-designed ovarian serous cancer patients with a long-term followup period up to 13 years. These proteomics results, which were confirmed by immunohistochemistry, support our hypothesis that $n m 23$ overexpression is a tumor progression marker. Furthermore, we found a greater overexpression of $n m 23$ in serous carcinomas than in mucinous tumors. This result contradicts previous reports of $n m 23$ immunoexpression in ovarian mucinous tumors. ${ }^{5}$ This discrepancy is likely due to the different interpretations and methods used in the studies. However, it should be noted that the immunohistochemistry of $n m 23$ should be interpreted cautiously as positive staining is restricted only to strong, diffuse expression not to focal, diffuse weak staining. ${ }^{6}$

The murine $n m 23$ cDNA was first identified by differential colony hybridization, which demonstrated its elevated expression in nonmetastatic murine K-1735 melanoma cell lines (nonmetastatic clone 23) and its reduced expression in a highly metastatic paired cell line. ${ }^{7}$ In humans, there are two nm23 genes, nm23-H1 and nm23-H2, that are located on chromosome band 17q-21.3. These genes encode human nucleoside diphosphate kinase A and B, respectively, ${ }^{8}$ and share significant homology with the Drosophila development gene awd and the Dictostelium NDP kinase gene. ${ }^{9}$ Expression levels of nm23 have been widely reported in human tumor cohorts. ${ }^{10}$ Although the expression of $\mathrm{nm} 23$ is not a universally independent predictive or prognostic factor for ovarian cancer, both metastasis suppression and disease progression have been linked to elevated $n m 23-H 1$ gene expression in different types of human tumors. As the simple overexpression of $n m 23$ is a poor prognostic factor, it is possible that nm23 plays some tissue-specific roles and that different mechanisms regulating its overexpression 

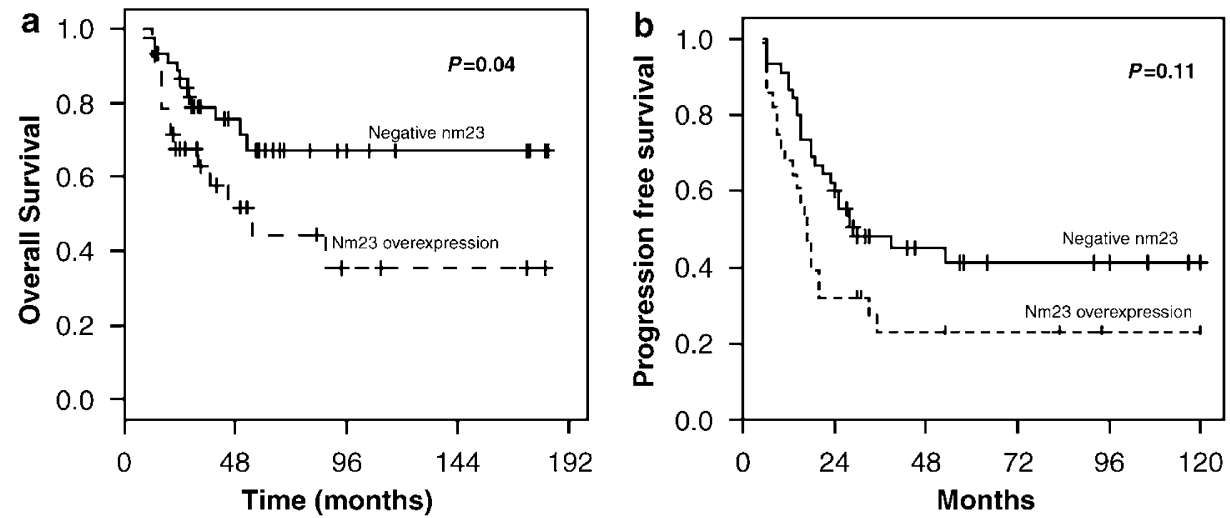

Figure 3 Kaplan-Meier survival with respect to $n m 23$ expression. (a) On overall survival analysis, nm23 overexpression was significantly associated with poor survival $(P=0.04)$. (b) The overexpression of $n m 23$ was significantly associated with poor progressionfree survival $(P=0.03)$. The continuous line represents the low expression of $n m 23$, and the dotted line represents the high expression of $n m 23$.

are involved in the development of certain types of tumors. Many studies have suggested an association between low nm23 expression and tumor aggressiveness and patient mortality in hepatoma, ${ }^{10}$ head and neck cancer, ${ }^{11,12}$ melanoma ${ }^{13}$ and breast cancer. ${ }^{14,15}$ Despite the widely accepted data on reduced nm23 expression and metastatic aggressiveness in breast cancer, it still does not represent an independent prognostic factor. ${ }^{6}$ Furthermore, there has been no correlation or positive association between $\mathrm{nm} 23$ expression and tumor aggressiveness reported in neuroblastoma, ${ }^{16}$ genitourinary tract cancers (including testis, prostate and endometrium), ${ }^{6}$ kidney cancer, thyroid cancer, ${ }^{17}$ pancreatic cancer ${ }^{18}$ or lung cancer. ${ }^{19}$ The role that the $n m 23-H 1$ gene plays in ovarian carcinoma has not yet been determined. The correlation between $\mathrm{nm} 23$ expression and ovarian cancer was first examined in a cohort study in $1994 .^{20}$ This study demonstrated that the overexpression of both $n m 23-H 1$ and $n m 23-H 2$ was associated with the progression of malignant tumors rather than with the progression of normal ovary or benign tumors. Subsequent studies, using other methods such as northern blotting or immunohistochemistry, confirmed $n m 23$ as a tumor progression marker. ${ }^{21-24}$ One report used immunohistochemistry to reveal a connection between nm23-H1 overexpression and both survival advantage and greater response to chemotherapy in 106 patients with advanced ovarian cancer. In this study, there were more nm23-H1-positive patients without lymph node involvement $(70 \%)$ than nm23-H1-positive patients with lymph node involvement $(40 \%){ }^{25}$ Later studies using allelic deletions of $17 \mathrm{q}$ revealed that an extremely high frequency of LOH (73-93\%) had no impact on the prognosis or metastatic behavior of ovarian cancer. ${ }^{21,22}$

Despite extensive research, the biological mechanisms by which elevated $n m 23$ expression suppresses metastasis are still not understood. The interaction between $n m 23-H 1$ and $K s r$ has been characterized, ${ }^{26}$ and it was shown that mutations that inactivate $K s r$ suppress the phenotypic effects of activated Ras. ${ }^{27}$ It is known that the overexpression of $n m 23-H 1$ results in diminished Map kinase activation, which is required for higher histidine protein kinase activity. ${ }^{28}$ Several characterized intracellular functions of $n m 23$, namely microtubule assembly and disassembly, ${ }^{29}$ signal transduction, ${ }^{30}$ transcription regulation ${ }^{31}$ and cellular adhesion, ${ }^{32}$ are known to influence certain metastatic processes.

In other biological contexts, increased nm23 expression correlates with aggressive disease. In these cases, the increased expression is associated with nm23 mutations, ${ }^{16,33}$ heterogeneity of phosphorylated isoforms, ${ }^{34}$ association with GTPaseactivating proteins ${ }^{35}$ and NDP kinase activity. ${ }^{36}$ The $n m 23$ mutations have seldom been detected in ovarian cancer. In a stratified univariate analysis and a complete multivariate model of 247 ovarian cancer patients, it was found that nm23 overexpression was an independent predictor of an ominous outcome. ${ }^{37}$ Schneider et al ${ }^{9}$ demonstrated that $n m 23$ overexpression had a negative impact on prognosis and that the $n m 23$ overexpression was to a wildtype $n m 23$ gene rather than to mutant variants. It is possible that nm23-H1 functions similarly to nm23H2, which has been shown in vitro to initiate transcription of c-myc, ${ }^{38}$ or another related oncogene associated with more malignant phenotypes in human ovarian cancer.

The present study to document that overexpression of $n m 23$ was significantly fewer in case of peritoneal seeding ( $27 \mathrm{vs} 60 \%$ ) represents that $n m 23$ expression suppresses peritoneal seeding. Regardless of peritoneal seeding suppression, however, $n m 23$ expression has shown that it was significantly associated with poor overall survival.

In conclusion, elevated $n m 23$ protein expression is an independent prognostic marker for diminished overall survival in patients with ovarian serous carcinoma. 


\section{Acknowledgements}

This study was supported by the CMB-YUHAN research fund (06-2006-0030; NHC) and the grant (no. HMP-A06-2598-A11018-06N1-00010A) of the 2006 Good Health R\&D Project, Ministry of Health \& Welfare, Korea.

\section{References}

1 Jemal A, Tiwari RC, Murray T, et al. Cancer statistics, 2004. CA Cancer J Clin 2004;54:8-29.

2 Naora H, Montell DH. Ovarian cancer metastasis: integrating insights from disparate model organisms. Nat Rev Cancer 2005;5:355-366.

3 Hibbs K, Skubitz KM, Pambuccian SE, et al. Differential gene expression in ovarian carcinoma: identification of potential biomarkers. Am J Pathol 2004;165: 397-414.

4 An HJ, Kim DS, Park YW, et al. Comparative proteomics of ovarian epithelial tumors. J Proteome Res 2006;5:1082-1090.

5 Tas F, Tuzlali S, Aydiner A, et al. Prognostic role of nm23 gene expression in patients with ovarian cancer. Am J Clin Oncol 2002;25:164-167.

6 Hartsough MT, Steeg PS. NM23/nucleoside diphosphate kinase in human cancers. J Biogenet Biomem 2000;32:301-308.

7 Stahl JA, Leone A, Rosengard AM, et al. Identification of a second human $n m 23$ gene, nm23-H2. Cancer Res 1991;51:445-449.

8 Steeg PS, Bevilacqua G, Kopper L, et al. Evidence for a novel gene associated with low tumor metastatic potential. J Natl Cancer Inst 1988;80:200-204.

9 Schneider J, Poll M, Jimez Z, et al. nm23-H1 expression defines a high-risk subpopulation of patients with early-stage epithelial ovarian carcinoma. Br J Cancer 2000;82:1662-1670.

10 Iizuka N, Oka M, Norma T, et al. NM23-H1 and NM23H2 messenger RNA abundance in human hepatocellular carcinoma. Cancer Res 1995;55:652-657.

11 Guo X, Min HQ, Zeng MS, et al. Nm23-H1 expression in nasopharyngeal carcinoma: correlation with clinical outcome. Int J Cancer 1998;79:596-600.

12 Lo Muzio LL, Mignogna MD, Pannone G, et al. The NM23 gene and its expression in oral squamous cell carcinoma. Oncol Rep 1999;6:747-751.

13 Florenes VA, Aamdal S, Myklebost O, et al. Levels of nm23 messenger RNA in metastatic malignant melanomas: inverse correlation to disease progression. Cancer Res 1992;52:6088-6091.

14 Barnes R, Masood S, Barker E, et al. Low nm23 protein expression in infiltrating ductal breast carcinomas correlates with reduced patient survival. Am J Pathol 1991;139:245-250.

15 Royds JA, Stephenson TJ, Rees RC, et al. NM23 protein expression in ductal in situ and invasive human breast carcinoma. J Natl Cancer Inst 1993; 85:727-731.

16 Leone A, Seeger RC, Hong CM, et al. Evidence for nm23 RNA overexpression, DNA amplification and mutation in aggressive childhood neuroblastomas. Oncogene 1993;8:855-865.

17 Arai T, Watanabe M, Onodera M, et al. Reduced nm23H1 messenger RNA expression in metasatic lymph nodes from patients with papillary carcinoma of the thyroid. Am J Pathol 1993;142:1938-1944.

18 Nakamori S, Ishikawa O, Ohigashi $\mathrm{H}$, et al. Clinicopathological features and prognostic significance of nucleoside diphosphate kinase/nm23 gene product in human pancreatic exocrine neoplasm. Int J Pancreatol 1993;14:125-133.

19 Engel M, Theisinger B, Seib T, et al. High levels of nm23-H1 and nm23-H2 messenger RNA in human squamous cell lung carcinoma are associated with poor differentiation and advanced tumor stages. Int J Cancer 1993;55:375-379.

20 Mandai M, Konishi I, Koshiyama M, et al. Expression of metastasis-related $n m 23-H 1$ and $n m 23-H_{2}$ genes in ovarian carcinomas: correlation with clinicopathology, EGFR, $c$-erbB-2, and $c$-erbB-3 genes, and sex-steroid receptor expression. Cancer Res 1994;54: 1825-1830.

21 Leary JA, Kerr J, Chenevix-Trench G, et al. Increased expression of the NMH1 gene is associated with metastasis in epithelial ovarian cancer. Int J Cancer 1995;64:189-195.

22 Viel A, Dall'Agnese L, Canzonieri V, et al. Suppressive role of the metastasis-related $n m 23-H 1$ gene in human ovarian carcinomas: association of high messenger RNA expression with lack of lymph node metastasis. Cancer Res 1995;55:2645-2650.

23 Schneider J, Romero H, Ruiz R, et al. NM23 expression in advanced and borderline ovarian carcinoma. Anticancer Res 1996;16:1197-1202.

24 Srivatsa PJ, Cliby WA, Keeney GL, et al. Elevated nm23 protein expression is correlated with diminished progression-free survival in patients with epithelial ovarian carcinoma. Gynecol Oncol 1996;60: 363-372.

25 Scambia G, Ferrandina G, Marone M, et al. Nm23 in ovarian cancer: correlation with clinical outcome and other clinicopathologic and biochemical prognostic parameters. J Clin Oncol 1996;14:334-342.

26 Hartsough M, Morrison D, Salerno M, et al. Nm23-H1 metastasis suppressor phosphorylation of kinase suppressor of Ras via a histidine protein kinase pathway. J Biol Chem 2002;277:32389-32399.

27 Kornfield K, Hom D, Horvitz H. The ksr-1 gene encodes a novel protein kinase involved in Rasmediated signaling in $C$. elegans. Cell 1995;83: 903-913.

28 Ouatas T, Salerno M, Palmieri D, et al. Basic and translational advances in cancer metastasis: Nm23. J Bioenerg Biomembr 2003;35:73-79.

29 Lakashmi MS, Parker C, Sherbet GV. Metastasis associated MTS1 and NM23 genes affect tubulin polymerization in B16 melanomas: a possible mechanism of their regulation of metastatic behavior of tumors. Anticancer Res 1993;13:299-303.

30 Liotta LA, Steeg PS. Clues to the function of nm23 and Awd proteins in development, signal transduction, and tumor metastasis provided by studies of Dictyostelium discoideum. J Natl Cancer Inst 1990;82: 1170-1172.

31 Postel EH. Cleavage of DNA by human NM23-H2/ nucleoside diphosphate kinase involves formation of a covalent protein-DNA complex. J Biol Chem 1999; 274:22821-22829.

32 Urano T, Furukawa K, Shiku H. Expression of nm23/ NDP kinase proteins on the cell surface. Oncogene 1993;8:1371-1376. 
33 Chang C, Zhu XX, Thoraval D, et al. Nm23-H1 mutation in neuroblastoma. Nature 1994;370:335-336.

34 MacDonald NJ, de la Rossa A, Benedict MA, et al. A serine phosphorylation of $n m 23$, and not its nucleoside diphosphate kinase activity, correlates with suppression of tumor metastatic potential. J Biol Chem 1993;268: 25780-25789.

35 Gilles AM, Presecan E, Vonica A, et al. Nucleoside diphosphate kinase from human erythrocytes. Structural characterization of the two polypeptide chains responsible for heterogeneity of the hexameric enzyme. J Biol Chem 1991;266:8784-8789.
36 Teng DH, Engele CM, Venkatesh TR. A product of the prune locus of Drosophila is similar to mammalian GTPase-activating protein. Nature 1991;353:437-440.

37 Mandai M, Konishi I, Komatsu T, et al. Mutation of the $n m 23$ gene, loss of heterozygosity at the nm23 locus and $K$-ras mutation in ovarian carcinoma: correlation with tumor progression and nm23 gene expression. Br J Cancer 1995;72:691-695.

38 Postel EH, Berberich SJ, Flint SJ, et al. Human $c-m y c$ transcription factor PuF identified as $\mathrm{nm} 23-\mathrm{H} 2$ nucleoside diphosphate kinase, a candidate suppressor of tumor metastasis. Science 1993;261:478-480. 